\title{
Ventricular Tachycardias in Structurally Normal Hearts - A Case Report and Review of the Literature

\author{
Juergen Schiefermueller
}

Papworth Hospital, Papworth Everard, Cambridge, United Kingdom

*Corresponding author: Juergen Schiefermueller, Papworth Hospital, Papworth Everard, 117 Vinery Rd, Flat 6, Cambridge, CB1 3DW, United Kingdom, Tel: +44(0)7951451208,E-mail: juergen@schiefermueller.com

\begin{abstract}
The acute management of broad complex tachycardias in the Emergency Department (ED) can often pose a significant challenge, not just for the more inexperienced of doctors. This is often due to the potentially lethal nature of this arrhythmia, the perceived complexity of ECG interpretation or handling of antiarrhythmic drugs. Whilst most doctors are aware that broad complex tachycardias are in the vast majority of cases related to structural abnormalities e.g. previous myocardial infarction, it is less well known that ventricular tachycardias can also occur in people with structurally normal hearts. Differentiation of the two is important as treatment is very different.
\end{abstract}

\section{Keywords}

Normal heart VT, Idiopathic VT, Benign VT

\section{Main Text}

About $90 \%$ of all broad complex tachycardias are of ventricular origin, this percentage increases to over $95 \%$ if there is a history of ischaemic heart disease (e.g. prior MI) or cardiomyopathy, i.e. a structural abnormality. This mere statistical fact can be used as a pre-test probability when dealing with patients presenting to the Emergency Department (ED) with broad complex tachycardias. Algorithms exist to differentiate between the causes of broad complex tachycardia, but they are often complex and not easily remembered. Heart rate or degree of hemodynamic compromise do not help differentiate between VT or SVT, contrary to common belief. Occasionally supraventricular rhythms (e.g. AV-(nodal)-re-entry-tachycardia $[A V(N) R T]$, atrial fibrillation/flutter or atrial tachycardia) can present with broad QRS duration, if there is aberrant conduction down to the ventricles due to pre-existing or rate-related (functional) bundle branch block, or pre-excitation due to an accessory pathway. Paced rhythms or artefacts are other reasons for wide QRS complexes.

Two cases of patients presenting with broad complex tachycardias are described here:

\section{Case 1}

A 31-year-old previously fit and well man presented to the emergency department (ED) with sudden onset palpitations and mild shortness of breath. This had started whilst out cycling competitively. He never had any similar symptoms before, his past medical and family histories were unremarkable, and he did not drink excessively or use illicit drugs. On examination he was apyrexial, hemodynamically stable, heart rate was 160 $\mathrm{bpm}$, blood pressure 120/70. The rest of the physical examination was unremarkable. His admission ECG is shown in Figure 1, demonstrating a regular monomorphic tachycardia with a QRS duration of $118 \mathrm{~ms}$, right bundle branch block morphology and left axis deviation. As per ALS guidelines he was treated with vagal manoeuvres and incremental doses of Adenosine for presumed SVT. This was unsuccessful, and the treating physician decided to arrange DC cardioversion. Whilst this was prepared, the patient reverted spontaneously to sinus rhythm.

\section{Case 2}

A 60-year-old man presented to ED with a 24-hour history of intermittent palpitations causing mild dizziness. His past medical history included "a procedure to his heart for palpitations" a few years prior, performed

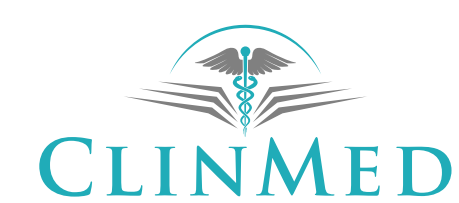

INTERNATIONAL LIBRARY

Citation: Schiefermueller J (2018) Ventricular Tachycardias in Structurally Normal Hearts - A Case Report and Review of the Literature. Int J Crit Care Emerg Med 4:036. doi.org/10.23937/2474-3674/1510036 Accepted: June 19, 2018: Published: June 21, 2018

Copyright: (C) 2018 Schiefermueller J. This is an open-access article distributed under the terms of the Creative Commons Attribution License, which permits unrestricted use, distribution, and reproduction in any medium, provided the original author and source are credited. 


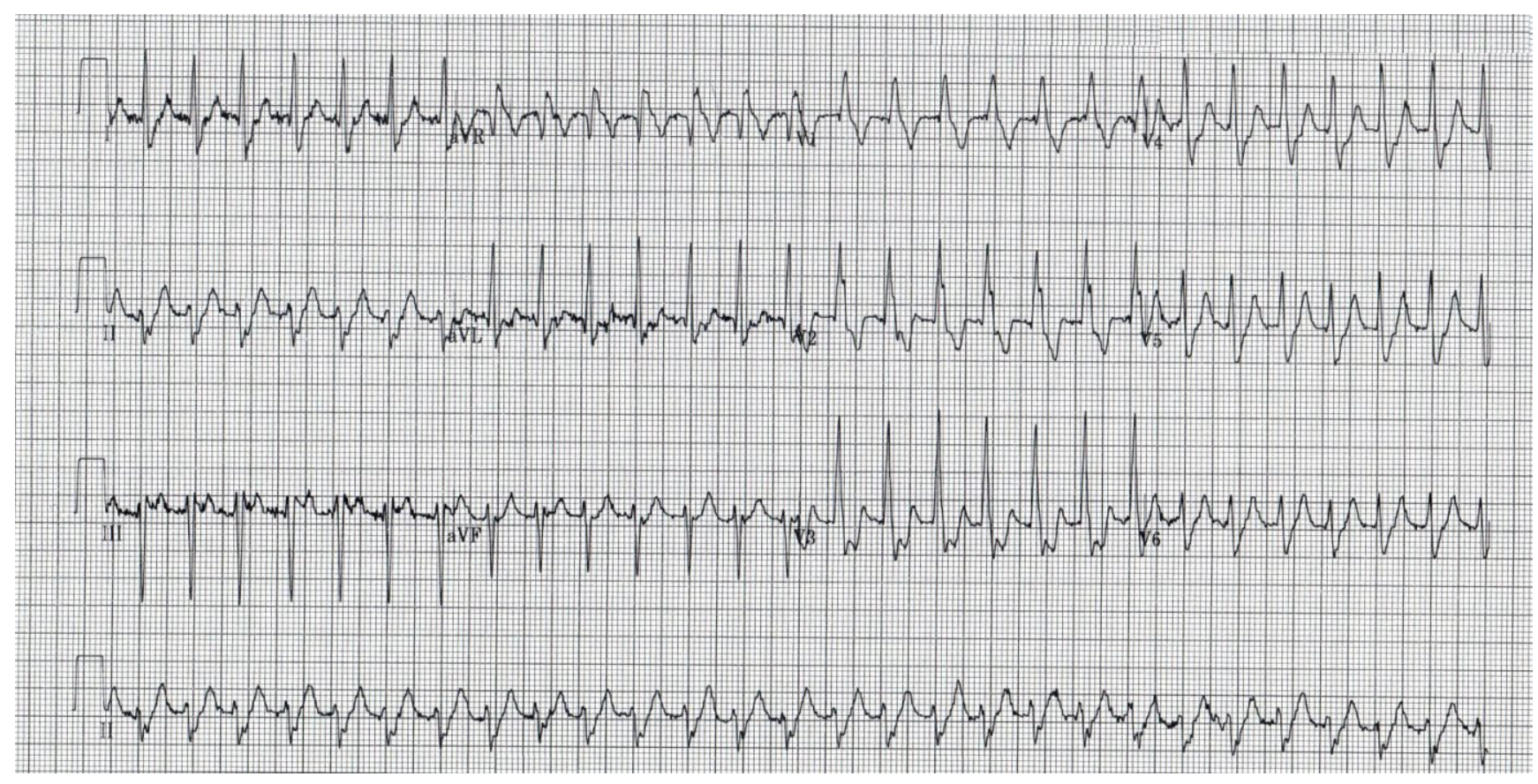

Figure 1: Fascicular VT: A regular monomorphic tachycardia with a QRS duration of $118 \mathrm{~ms}$, right bundle branch block morphology and left axis deviation.

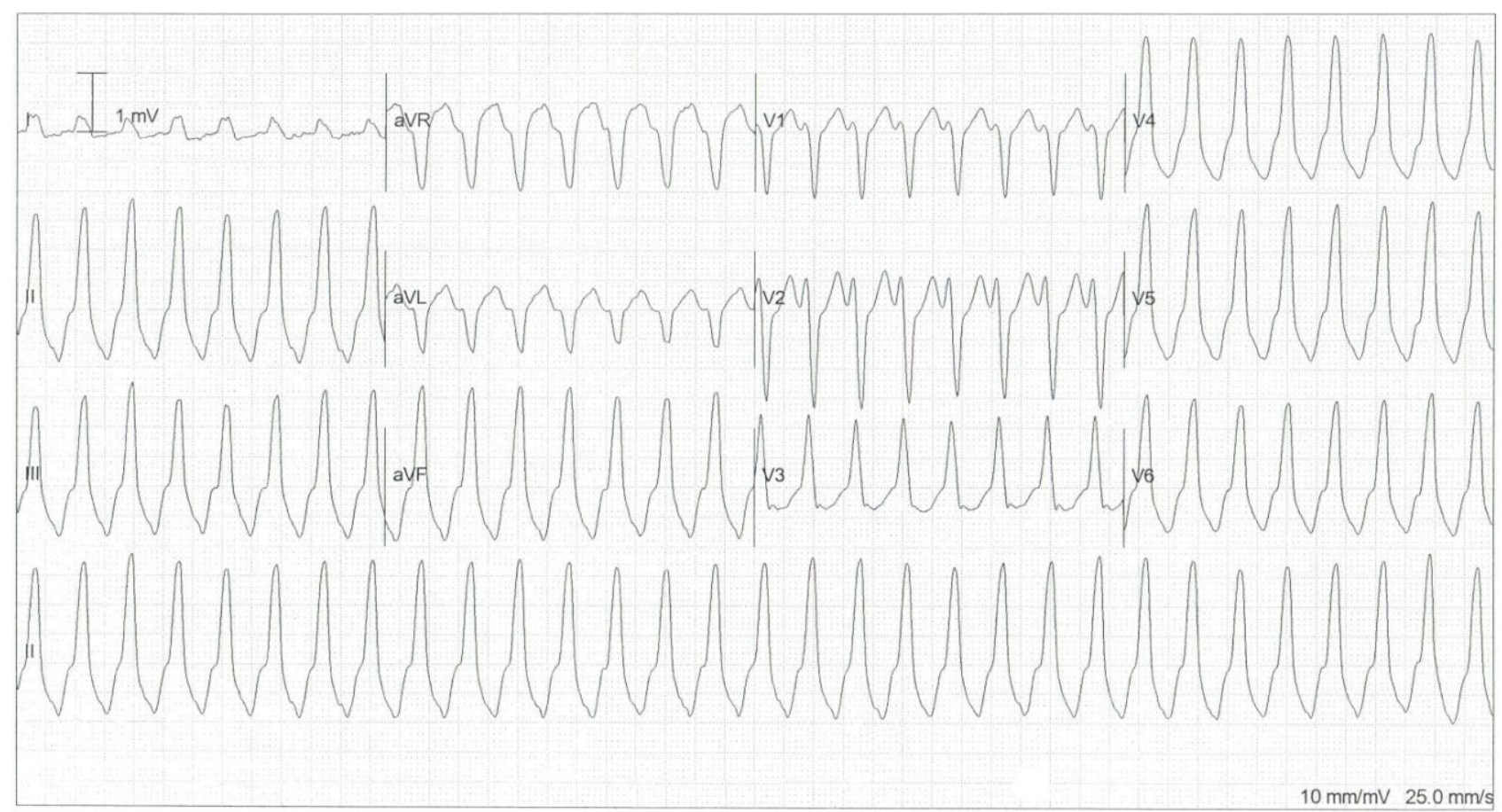

Figure 2: Right ventricular outflow tract (RVOT)-VT: A regular broad complex tachycardia with inferior axis and left bundle branch block morphology.

at a different hospital. He did not remember the exact details. In the meanwhile, he had moved to house and was lost to follow-up. On examination he appeared well, clinical examination was unremarkable. Vital signs showed a heart rate of $180 \mathrm{bpm}$, blood pressure was $100 / 80$. His ECG is shown in Figure 2, demonstrating a regular broad complex tachycardia with inferior axis (QRS positive in leads II, III, aVF and negative in $\mathrm{QVL}$ ) and left bundle branch block morphology. He was given 8 mmol magnesium and a bolus of $300 \mathrm{mg}$ intravenous
Amiodarone but required emergency DC cardioversion when his blood pressure dropped to $70 / 40$ a few minutes into the Amiodarone infusion. Over the next 24 hours he had further non-sustained runs of broad complex tachycardia with a similar morphology.

\section{Discussion}

It is less well known that there is a subset of ventricular tachycardias that occur in people with structurally normal hearts. These arrhythmias have a benign/excel- 
lent prognosis, and are often called "idiopathic VTs", "benign VTs" or "normal heart VTs". Reports of sudden cardiac deaths are extremely rare. Treatment options include medication or catheter ablation. The two most common syndromes have been presented here, fascicular tachycardia in case 1 , and right ventricular outflow tract tachycardia (RVOT-VT) in case 2.

Fascicular (ventricular) tachycardias most commonly arise from foci in the left posterior fascicle of the left bundle branch, likely due to a re-entrant circuit there. They usually occur in young males, often triggered by stress or exertion. They are often misdiagnosed as SVTs instead of VTs (as in the case presented) due to their relatively narrow QRS duration, though the right bundle branch block morphology with left axis deviation reveal their origin to be in the left posterior area of the heart. They are not responsive to Adenosine but will ordinarily respond to Verapamil. Accordingly, they are also known as "Verapamil-sensitive idiopathic left ventricular tachycardia" [1]. Patients can be maintained on calcium channel blockers, but radiofrequency ablation is possible and offers a permanent cure. The patient discussed in the above case underwent this procedure and has since been symptom free.

Right ventricular outflow tract (RVOT) tachycardias originate from cells around the RVOT [2]. Left ventricular outflow tract VT exists, but is rarer. Similar to fascicular VTs the paroxysms are often triggered by exercise, then characteristically causing bouts of sustained VT. Often symptoms like palpitations are mild and syncope or sudden cardiac death is rare. The ECG pattern characteristically is of a left bundle branch block appearance with strongly inferior axis (positive QRS in leads II, III and aVF). Commonly frequent ventricular ectopy, couplets or salvos of non-sustained ventricular tachycardias are seen. The tachycardia is characteristically sensitive to iv Adenosine, and symptoms are usually well controlled long-term with beta-blockers, calcium channel blockers or type IC antiarrhythmics (e.g. Flecainide). Ablation of the focus is a possible long-term curative option for patients that remain symptomatic. The main differential diagnosis for LBBB/inferior axis tachycardias is arrhythmogenic right ventricular cardiomyopathy/dysplasia (ARVC/D). VTs from this condition don't usually terminate with Adenosine. Often the characteristic ECG pattern of RVOT-ventricular tachycardias are not recognized and patients are treated as per ALS guidelines with Amiodarone. The latter, apart from achieving cardioversion in only about $20 \%$ of cases in the first hour [3], often causes hypotension itself, mistaken as hemodynamical deterioration from the tachycardia and leading to (unnecessary) DC-cardioversion. In this case obtaining the patients old notes revealed that the procedure he was referring to was a prior RVOT-tachycardia ablation. His maintenance beta blockers were increased which lead to suppression of his symptoms, and he was referred for redo-ablation as an outpatient.

In summary, patients presenting with broad complex tachycardias can with over $90 \%$ certainty assumed to be in ventricular tachycardia, especially if there is a history of ischaemic heart disease/previous MI [4]. Standard ALS guidelines should be followed when treating these cases. The experienced physician should be aware though that "normal heart VTs" do in fact exist, and ideally be able to recognize and treat or refer them accordingly [5].

\section{Conflict of Interest}

None declared.

\section{Acknowledgements, Competing Interests, Fund- ing}

None.

\section{References}

1. Belhassen B, Shapir I, Pelleg A, Copperman I, Kauli N, et al. (1984) Idiopathic recurrent sustained ventricular tachycardia responsive to verapamil: An ECG-electrophysiologic entity. Am Heart J 108: 1034-1037.

2. Zipes DP, Camm AJ, Borggrefe M, Buxton AE, Chaitman $B$, et al. (2006) ACC/AHA/ESC 2006 guidelines for management of patients with ventricular arrhythmias and the prevention of sudden cardiac death: A report of the American College of Cardiology/American Heart Association Task Force and the European Society of Cardiology Committee for Practice Guidelines (Writing Committee to Develop guidelines for management of patients with ventricular arrhythmias and the prevention of sudden cardiac death) developed in collaboration with the European Heart Rhythm Association and the Heart Rhythm Society. Europace 8: 746-837.

3. Tomlinson DR, Cherian P, Betts TR, Bashir Y (2008) Intravenous amiodarone for the pharmacological termination of haemodynamically-tolerated sustained ventricular tachycardia: Is bolus dose amiodarone an appropriate first-line treatment? Emerg Med J 25: 15-18.

4. Steinman RT, Herrera C, Schuger CD, Lehmann MH (1989) Wide QRS tachycardia in the conscious adult: Ventricular tachycardia is the most frequent cause. JAMA 261: 10131016.

5. Akhtar M (1990) Clinical spectrum of ventricular tachycardia. Circulation 82: 1561-1573. 